\title{
Approach to Ideal Network Formation in Free-Radical Crosslinking Copolymerization of Benzyl Acrylate with 1,6-Hexanediol Diacrylate
}

\author{
Akira Matsumoto ${ }^{\dagger}$ and Akira TANiguchi \\ Department of Applied Chemistry, Faculty of Engineering and \\ High Technology Research Center, Kansai University, \\ Suita, Osaka 564-8680, Japan
}

(Received March 8, 1999)

KEY WORDS Benzyl Acrylate/1,6-Hexanediol Diacrylate / Ideal Network Formation / Crosslinking/
Gelation /

We have been concerned with the mechanism of the three-dimensional network formation in the free-radical polymerization of multivinyl compounds with the intention of elucidating the crosslinking reaction mechanism and, moreover, controlling the network formation to molecular-design vinyl-type network polymers with high performance and functionality. Thus the experimental results obtained mainly in our laboratory have recently been reviewed, ${ }^{1}$ especially focusing on clarification of the mechanistic reasons for the greatly delayed gelation from Flory-Stockmayer gelation theory (F-S theory). ${ }^{2}$

Dotson et al. ${ }^{3}$ and Zhu et al. ${ }^{4}$ demonstrated that the classical F-S theory is inapplicable and, moreover, an alternative theory should be sought. Our interpretation of the greatly delayed gelation suggests that the actual gel point should be close to the theoretical one if the experiment is done under the polymerization conditions in which the polymer concentration, at the theoretical gel point, is high enough to reduce the significance of a thermodynamic excluded volume effect as a primary factor, and the intramolecular crosslinking preceded by the intermolecular crosslinking could be suppressed.

We attempted to verify our understanding of the crosslinking reaction mechanism of monovinyl-divinyl copolymerization: In previous articles ${ }^{5,6}$ the ratio of the actual gel point to the theoretical one reached 1.3, 1.05, or 1.06 , close to unity, in the copolymerizations of methyl methacrylate with $0.03 \mathrm{~mol} \%$ of ethylene dimethacrylate, ${ }^{5}$ butylene dimethacrylate, or nonapropyleneglycol dimethacrylate, ${ }^{6}$ respectively, demonstrating the good applicability of $\mathrm{F}-\mathrm{S}$ theory. To extend this discussion, this article deals with the free-radical crosslinking copolymerization of benzyl acrylate (BzA) with 1,6hexanediol diacrylate (HDA) since polyacrylate backbone is more flexible than polymethacrylate and the polymer chain flexibility is related to the occurrence of intramolecular crosslinking reaction ${ }^{7}$ as a secondary factor for greatly delayed gelation from theory. ${ }^{1}$

\section{EXPERIMENTAL}

BzA and HDA, supplied by Kyoeisha Chemical Co.,

\footnotetext{
† To whom correspondence should be addressed
}

Ltd., as monomers, and 1,1'-azobiscyclohexane-1-carbonitrile $(\mathrm{ACHCN})$ as initiator were purified by conventional methods. Lauryl mercaptan (LM) as chain transfer agent was used as commercially available, extra pure reagent.

Polymerization was carried out as described previously. ${ }^{8}$ After a predetermined time of reaction, the copolymer was precipitated by pouring the reaction mixture into a large excess of chilled methanol containing a small amount of hydroquinone as inhibitor. Purification of the polymer was done by reprecipitation from a tetrahydrofuran (THF)-precipitant system. The gel fraction of polymer at conversions beyond the gel point was separated by extracting the sol fraction with THF.

Weight-average molecular weights, $\bar{M}_{w}$, and molecular-weight distribution (MWD) were measured by sizeexclusion chromatography (SEC) using a dual detector system, set in the direction of flow, consisting of a multiangle laser light scattering (MALLS) device and differential refractometer in sequence. SEC-MALLS measurements were carried out at $40^{\circ} \mathrm{C}$ in THF using a Shodex GPC KF-806L $\times 5$ columns, at polymer concentrations $0.1-0.5 \%(\mathrm{w} / \mathrm{v})$ and flow rate $1 \mathrm{~mL} \mathrm{~min}^{-1}$, and $\bar{M}_{w}$ was calculated from Zimm plots. The MALLS device was a DAWN Model F (Wyatt Technology Corp.) whose laser beam, wavelength $632.8 \mathrm{~nm}$, was focused on a $67 \mu \mathrm{L}$ flow cell.

\section{RESULTS AND DISCUSSION}

Applicability of $F-S$ Theory as Comparison of Actual Gel Point with Theoretical One

As in previous work, ${ }^{5,6}$ the copolymerization of BzA was carried out with a small amount of HDA as crosslinker at a high monomer concentration because under this condition the polymer concentration at the theoretical gel-point conversion becomes high and the content of pendant vinyl groups in the prepolymer is quite small. As a consequence, the thermodynamic excluded volume effect and intramolecular crosslinking were suppressed. A small amount of LM as a chain transfer agent was added to keep the primary chain length constant during polymerization. The commonly observed Trommsdorff effect or gel effect, ${ }^{9,10}$ enlarging the primary chain length with conversion, is suppressed by 
the addition of a chain transfer agent.

Figure 1 shows the conversion-time curves for the solution copolymerization of $\mathrm{BzA}$ with $0.03 \mathrm{~mol} \%$ of HDA in 1,4-dioxane at a dilution of $2 / 3$ using 0.01 mol L-1 of $\mathrm{ACHCN}$ at $50^{\circ} \mathrm{C}$ in the presence of $4.2 \times 10^{-4} \mathrm{M}$ of LM. The percentage of gel polymer obtained by the sol-gel separation is plotted against time in Figure 1. The actual gel point was determined by extrapolating the gel formation curve to zero percentage. The conversion at which gel starts to form, i.e., the gel point, was estimated to be $82.0 \%$. In addition, no gel effect was observed, although the polymerization was accompanied by gelation.

Figure 2 shows the dependence of $\bar{M}_{w}$ on conversion; the primary chain length $\bar{P}_{\mathrm{w}, 0}$ was estimated by the

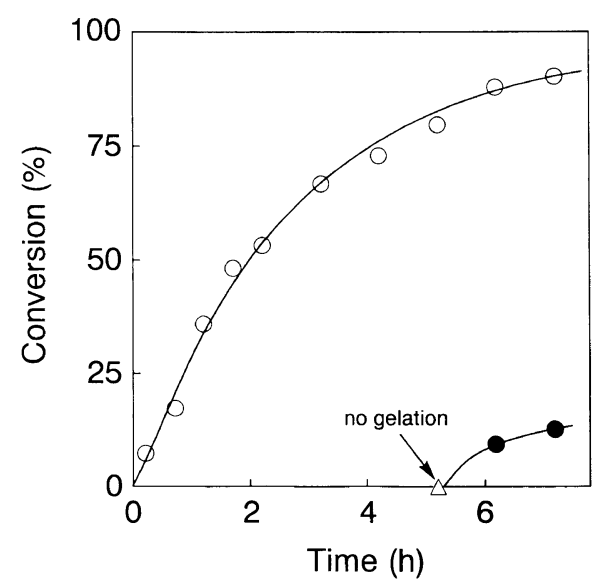

Figure 1. Conversion-time curve for solution copolymerization of $\mathrm{BzA}$ with $0.03 \mathrm{~mol} \%$ of HDA. Polymerization was conducted in 1,4-dioxane at a dilution of $2 / 3$ using $0.01 \mathrm{M}$ of $\mathrm{ACHCN}$ at $50^{\circ} \mathrm{C}$ in the presence of $4.2 \times 10^{-4} \mathrm{M}$ of LM. Open and full symbols are total and gel polymers, respectively.

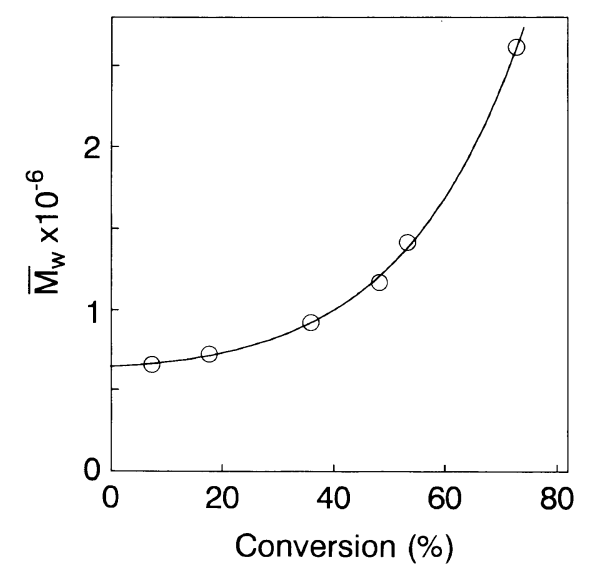

Figure 2. Dependence of $\bar{M}_{w}$ on conversion (see Figure 1). extrapolation of the curve to zero conversion. $\bar{P}_{\mathrm{w}, 0}$ was used to calculate the theoretical gel point according to Stockmayer's equation ${ }^{2}: \alpha_{\mathrm{c}}=(1 / \rho)\left(\bar{P}_{\mathrm{w}, 0}-1\right)^{-1}$, where $\rho$ is the fraction of all double bonds residing on divinyl units in the initial system.

Table I summarizes the results of a comparison of actual gel point with theoretical one for the copolymerizations of BzA with $0.03,0.06$, and $0.5 \mathrm{~mol} \%$ of HDA in the presence of an appropriate amount of LM. In the presence of 0.06 and $0.03 \mathrm{~mol} \%$ of HDA, the ratios of the actual gel points to the theoretical ones reached 1.7 and 1.9 , respectively, close to unity as compared with well-known cases where gelation occurs frequently $1-2$ orders of magnitude less than predicted, demonstrating the good applicability of F-S theory. Here it should be noted that for BzA/HDA (99.97/0.03) copolymerization a slightly higher value of 1.9 was observed than the value of 1.7 for BzA/HDA (99.94/0.06) copolymerization. This may be due to a higher primary chain length (see Table I) as the delay of gelation from theory became smaller for shorter primary chain length. ${ }^{1}$

\section{MWD Curves Governed by F-S Theory}

The F-S theory suggests that the MWD curves should rapidly broaden towards a higher-molecular-weight side with conversion as a result of exclusive intermolecular crosslinking reaction leading to ideal network formation governed by theory. ${ }^{2,11}$ SEC is commonly used to determine molecular weight and MWD of prepolymers. However, as a technique it is not always applicable to our crosslinking polymerization systems because the structure of prepolymer changes from a linear to a branched form with the progress of polymerization and the content of ring or loop structure introduced into the linear portion or primary chain and content of multiple crosslinkage in the branched form depend on the polymerization conditions. SEC-MALLS is expected to be very useful and efficient in characterizing our prepolymers because the use of MALLS, as an absolute detector, enables the molecular weight and MWD to be determined directly without any calibration. ${ }^{6}$

Figure 3 shows variation of MWD curves with conversion in the copolymerization of $\mathrm{BzA}$ with 0.5 and $0.06 \mathrm{~mol} \%$ of HDA as ratios of the actual gel points to the theoretical ones were 5.6 and 1.7, respectively. For the latter copolymerization, more tailed curves were observed with conversion as a reflection of the exclusive occurrence of intermolecular crosslinking reaction required for an ideal network formation governed by F-S theory.

The resulting prepolymers characterized as precursors of ideal network-polymers are clearly evident in Figure

Table I. Comparison of actual and theoretical gel points in the copolymerization of BzA with HDA ${ }^{\mathrm{a}}$

\begin{tabular}{|c|c|c|c|c|c|}
\hline \multirow{2}{*}{$\begin{array}{l}\text { HDA } \\
\mathrm{mol} \%\end{array}$} & \multirow{2}{*}{$\frac{\mathrm{LM}}{10^{-3} \mathrm{M}}$} & \multirow{2}{*}{$\bar{P}_{\mathrm{w}, 0} \times 10^{-3 \mathrm{~b}}$} & \multicolumn{2}{|c|}{ Gel point $/ \%$} & \multirow{2}{*}{$\begin{array}{c}\text { Actual gel point }{ }^{\mathrm{d}} \\
\text { Theoretical gel point }\end{array}$} \\
\hline & & & Theoretical $^{\mathrm{e}}$ & Actual $^{\mathrm{c}}$ & \\
\hline 0.5 & 1.5 & 2.52 & 3.98 & $23.5(23.3)^{\mathrm{d}}$ & 5.9 \\
\hline 0.06 & 2.0 & 1.76 & 47.5 & $87.0(86.9)^{\mathrm{d}}$ & 1.8 \\
\hline 0.03 & 0.42 & 3.95 & 42.2 & $82.0(81.9)^{\mathrm{d}}$ & 1.9 \\
\hline
\end{tabular}

${ }^{\mathrm{a}}$ In dioxane, dilution $2.3,[\mathrm{ACHCN}]=0.01 \mathrm{M}, 50^{\circ} \mathrm{C} . \quad{ }^{\mathrm{b}}$ Estimated by SEC-MALLS. ${ }^{\mathrm{c}}$ Obtained on monomer basis. ${ }^{\mathrm{d}}$ Obtained as the vinyl group conversion calculated by assuming equal reactivity of BzA and HDA vinyl groups. ${ }^{\mathrm{e}}$ Theoretical gel point: $\alpha_{\mathrm{c}}=(1 / \rho)\left(\bar{P}_{\mathrm{w}, 0}-1\right)^{-1}$. 


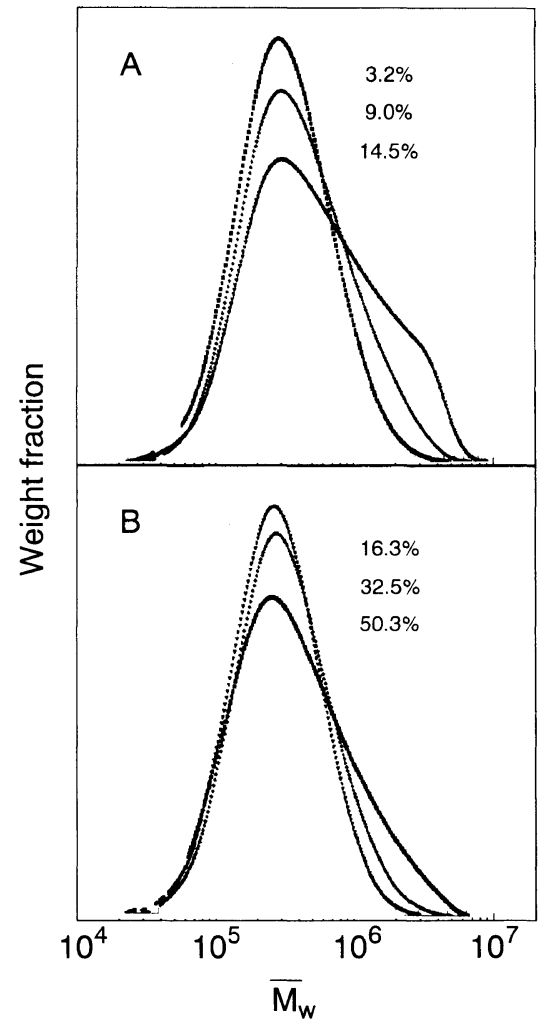

Figure 3. Variation of MWD curves with conversion for solution copolymerization of BzA with (A) 0.5 and (B) $0.06 \mathrm{~mol} \%$ of HDA (see Figure 1).

4; a roughly single correlation curve of molecular weight versus elution volume was obtained regardless of conversion, consistent with the above discussion. The slope of the correlation curve of methyl methacrylate-ethylene dimethacrylate $(90: 10)$ copolymer became steeper, indicating intramolecular cyclization and intramolecular crosslinking which result reduced molecular size. ${ }^{6}$

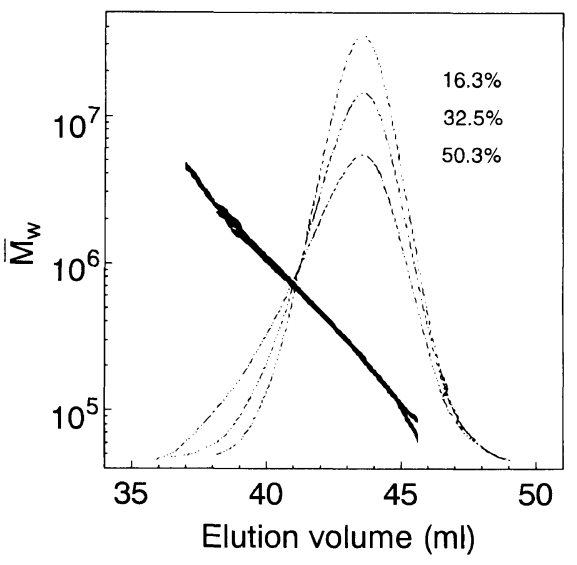

Figure 4. Variation of RI-monitored SEC curves and correlation of molecular weight versus elution volume with conversion for solution copolymerization of BzA with $0.06 \mathrm{~mol} \%$ of HDA (see Figure 1).

\section{REFERENCES}

1. A. Matsumoto, Adv. Polym. Sci., 123, 41 (1995).

2. W. H. Stockmayer, J. Chem. Phys., 12, 125 (1944).

3. N. A. Dotson, C. W. Macosko, and M. Tirrell, in "Synthesis, Characterization, and Theory of Polymeric Networks and Gels," Plenum Press, New York, N.Y., 1992, p 319.

4. S. Zhu and A. E. Hamielec, Macromol. Chem., Macromol. Symp., 63, 247 (1993)

5. A. Matsumoto, S. Okuno, and H. Aota, Macromol. Symp., 93, 1 (1995).

6. A. Matsumoto, A. Okamoto, S. Okuno, and H. Aota, Angew. Makromol. Chem., 240, 275 (1996).

7. A. Matsumoto, Y. Hasei, and H. Aota, Netsukokasei Jushi ( J. Thermoset. Plast. Jpn.), 15, 117 (1994).

8. A. Matsumoto and M. Oiwa, J. Polym. Sci., A-1, 8, 751 (1970).

9. E. Trommsdorff, H. Kohle, and P. Lagally, Makromol. Chem. 1, 169 (1948).

10. K. Horie, A. Otagawa, M. Muraoka, and I. Mita, J. Polym. Sci., Polym. Chem. Ed., 13, 445 (1975).

11. M. Oiwa, Nippon Kagaku Zasshi, 76, 684 (1955). 